\title{
Corrigendum: A Single-Cell Sequencing Guide for Immunologists
}

\author{
Peter See ${ }^{1}$, Josephine Lum $^{1}$, Jinmiao Chen ${ }^{1 *}$ and Florent Ginhoux ${ }^{1,2 *}$ \\ ${ }^{1}$ Singapore Immunology Network, Agency for Science, Technology and Research, Singapore, Singapore, ${ }^{2}$ Shanghai Institute \\ of Immunology, Shanghai JiaoTong University School of Medicine, Shanghai, China
}

Keywords: single-cell RNA sequencing, MARS-seq, SMART-seq, fluidigm C1, 10X genomics chromium, immunology, dendritic cells

\section{A Corrigendum on}

A Single-Cell Sequencing Guide for Immunologists

by See, P., Lum, J., Chen, J., and Ginhoux, F. (2018). Front. Immunol. 9:2425. doi: 10.3389/fimmu.2018.02425

In the original article, there was an error. The statement "Human peripheral blood consists of $\sim 90 \%$ lymphocytes, $10 \%$ monocytes and $1 \%$ dendritic cells" is incorrect.

A correction has been made to the Case study: Using scRNA-seq to resolve dendritic cell ontogeny:

\section{OPEN ACCESS}

Edited and reviewed by:

Shalin Naik,

Walter and Eliza Hall Institute of

Medical Research, Australia

*Correspondence:

Florent Ginhoux

florent_ginhoux@

immunol.a-star.edu.sg

Jinmiao Chen

chen_jinmiao@immunol.a-star.edu.sg

Specialty section:

This article was submitted to

Antigen Presenting Cell Biology,

a section of the journal

Frontiers in Immunology

Received: 21 December 2018 Accepted: 01 February 2019

Published: 26 February 2019

Citation:

See P, Lum J, Chen J and Ginhoux F (2019) Corrigendum: A Single-Cell

Sequencing Guide for Immunologists.

Front. Immunol. 10:278.

doi: 10.3389/fimmu.2019.00278
"A cell type of interest as a case study for this review is Dendritic Cell (DC) as it is small in numbers and heterogeneous in subsets (48). Human peripheral blood mononuclear cells consist of approximately $90 \%$ lymphocytes, $10 \%$ monocytes, and $1 \%$ dendritic cells. In a recent report, scRNA-seq using the 10X Genomics Chromium system was performed on 68,000 unsorted peripheral blood mononuclear cells (PBMC) in order to identify various immune cell populations (7). While this study was able to identify all the major immune cell populations present in blood, the authors found it difficult to identify or resolve cell types whose frequency was less than $1 \%$. Although this type of approach can provide a useful snapshot of the cellular composition of a given tissue, it may be necessary to enrich rare cell types in the sample prior to scRNAseq, for example by pre-sorting using known or novel surface markers. Indeed, this strategy was recently used by two separate groups to identify human precursors of dendritic cells (pre-DC) in human peripheral blood $(8,10)$. Villani and colleagues focused on lineage ${ }^{-} \mathrm{HLA}_{-} \mathrm{DR}^{+}$cells, which comprise known blood DCs and monocytes (8). In their study, the authors performed SMART-seq 2 on 2,400 lineage ${ }^{-} \mathrm{HLA}^{-\mathrm{DR}^{+}}$single cells and detected transcriptionally distinct cell clusters that could be identified using novel surface markers, thus facilitating their isolation by FACS and subsequent analysis by scRNA-seq to validate transcriptional identity. With this method, the authors were able to identify several new types of DCs and monocytes as well as a novel DC precursor population. Separately, our group focused on human blood lineage ${ }^{-}$HLA-DR ${ }^{+}$CD $135^{+}$ cells which consist of both DC subsets and their precursors (10). We performed MARS-seq on 710 lineage ${ }^{-} \mathrm{HLA}_{-} \mathrm{DR}^{+} \mathrm{CD}_{135^{+}}$single cells and identified two transcriptionally distinct clusters of plasmacytoid DC (pDC), two subpopulations of conventional DC (cDC), and a new cluster that was later found to constitute pre-DC. Further interrogation of this novel pre-DC population in human bone marrow and peripheral blood revealed that the pre-DC compartment contained distinct lineage-committed sub-populations (one early "uncommitted" CD123 high pre-DC subset, and two $\mathrm{CD} 45 \mathrm{RA}^{+} \mathrm{CD}_{12} 3^{\text {low }}$ lineage-committed subsets with distinct functional features). Together, these 
studies demonstrate that different scRNA-seq platforms can be successfully applied to similar biological questions in complementary ways."

The authors apologize for this error and state that this does not change the scientific conclusions of the article in any way. The original article has been updated.
Copyright (c) 2019 See, Lum, Chen and Ginhoux. This is an open-access article distributed under the terms of the Creative Commons Attribution License (CC BY). The use, distribution or reproduction in other forums is permitted, provided the original author(s) and the copyright owner(s) are credited and that the original publication in this journal is cited, in accordance with accepted academic practice. No use, distribution or reproduction is permitted which does not comply with these terms. 